\title{
Ulcerative Uremic Stomatitis: Canary in a Coalmine
}

\author{
Dr. Latika Bachani ${ }^{1 *}$, Dr. Ashok L. ${ }^{2}$, Dr. Monika Singh ${ }^{3}$, Dr. Srishti Kumar ${ }^{4}$, Dr. Tanvi Tiwari ${ }^{5}$ \\ ${ }^{1}$ Assistant Professor, Department of Oral Medicine and Radiology, Faculty of Dental Sciences, SGT university, Gurugram-122001, Haryana, India \\ ${ }^{2}$ Prof and Head, Dept. of Oral Medicine and Radiology, Bapuji Dental College and Hospital, Davanagere, Karnataka 577004, India \\ ${ }^{3}$ Post graduate, Department of Oral Medicine and Radiology, Faculty of Dental Sciences, SGT university, Gurugram-122001, Haryana, India \\ ${ }^{4}$ General Dentist, Faculty of Dental Sciences, SGT University, Gurugram-122001, Haryana, India \\ ${ }^{5}$ General Dentist, Faculty of Dental Sciences, SGT university, Gurugram-122001, Haryana, India
}

DOI:10.36348/SJM.2019.v04i09.002

| Received: 15.07.2019 | Accepted: 22.07.2019| Published: 20.09.2019

*Corresponding author: Dr. Latika Bachani

\section{Abstract}

It is a well-known verity that many systemic diseases are manifested in the oral cavity. Irrespective of the organ system involved, changes frequently occur in the oral cavity reflecting disease elsewhere in the body. Uremic stomatitis is one such rare oral mucosal disorder associated with end-stage renal failure. Clinically it is characterized by the presence of painful plaques and crusts that are usually distributed on the buccal mucosa, dorsal or ventral surface of the tongue, gingiva, lips, and floor of the mouth. Hence, it is accurately said that oral cavity is the mirror of the general health and treating the underlying disease first followed by local therapy is the way to manage these conditions. Here, we discuss a rare case of a young male patient presenting with oral manifestations of previously undiagnosed renal failure.

Keywords: Uremic Stomatitis, Uremia, Ulcerative Uremic Stomatitis, End-Stage Renal Failure.

Copyright @ 2019: This is an open-access article distributed under the terms of the Creative Commons Attribution license which permits unrestricted use, distribution, and reproduction in any medium for non-commercial use (NonCommercial, or CC-BY-NC) provided the original author and source are credited.

\section{INTRODUCTION}

Man is a mosaic of many tissues and organs, each one functioning and metabolizing in its own peculiar way. Each organ has a role in the maintenance of body homeostasis, specially the kidney which is responsible for excretory functions and acid base electrolyte balance along with blood pressure regulation and endocrine functions. Nephrons, which are about 1 million per kidney, are the functional unit of kidney. These cells are incapable of regeneration. Renal function is maintained until approximately half of the nephrons are destroyed. The progressive loss of kidney function, caused by various pathologic disorders, results in a condition known as uremia. It is the clinical and laboratory syndrome reflecting the dysfunction of all organ systems as a result of untreated or under-treated acute or chronic renal failure [1]. Chronic renal failure (CRF) affects a range of tissues and systems, leading to complications of nervous, cardiovascular, respiratory, endocrine, hematopoietic, gastrointestinal, urological, skin, mucosa and craniofacial complex [2].

The commonly noticed oral manifestations in these patients are xerostomia, uremic fetor,dysgeusia, gingival inflammation, pallor of the oral mucosa, ecchymosis, petechiae, and hemorrhages,stomatitis, mucositis, glossitis and burning sensation of oral cavity. Such patients are more prone to bacterial and candidial infections [3]. The occurrence of gingivitis is comparatively low because uremia alters the inflammatory response to bacterial plaques in gingival tissue. Elevated salivary $\mathrm{pH}$,decreased salivary magnesium, high levels of salivary urea and phosphorus lead to precipitation of calcium-phosphorus and calcium oxalate, and, thus, dental calculus formation'The incidence of dental caries, on the contrary is low because of highly buffered and alkaline saliva [4].The primary and permanent dentition, particularly canines show enamel hypoplasia. Narrowing or calcification of the tooth pulp chamber and delayed eruption of the permanent teeth are other noticeable findings in these patients [5].

\section{CASE REPORT}

A 24 years old male patient reported to us with a chief complaint of painful ulcers in his mouth since 8 days. History of presenting illness revealed that the ulcers were insidious in onset, progressive in severity and size. They were associated with pain and bleeding. The patient experienced difficulty in mastication, swallowing and drinking water due to that. It is also associated with alteration in taste, foul breath and weight loss. There were no prodromal symptoms associated. It is the first episode of such ulcers in the oral cavity and no such lesions were noted anywhere else on the body. His past medical and dental history 
was normal and family history was non-significant. The patient was married, followed mixed diet, and had habit of chewing tobacco occasionally and was nonalcoholic.

On general physical examination, his gait was normal. He was moderately built and nourished, slightly disoriented with slurred speech and was afebrile. Diffuse blackish pigmentation was noted on the skin over the neck (Fig.1). Fingernails had a pinkish red band occupying $30 \%$ of the nail bed distally and a white band proximally which was a classical presentation of Lindsay's lines (Fig.2). Pedal edema was also noted. There was generalized pallor and dryness of the skin.

Further on extraoral examination, he presented with generalised pallor associated with yellowish tinge and dryness of facial skin, periorbital edema, incompetent and everted lower lip (Fig.3). Right and left submandibular lymph nodes were palpable. They were solitary, roughly oval in shape, measuring approx. $5 \mathrm{~mm}$ in diameter, firm, tender, mobile. Mouth opening was restricted and associated with pain. There was foul smell resembling that of a rotten fruit noted intra-orally.

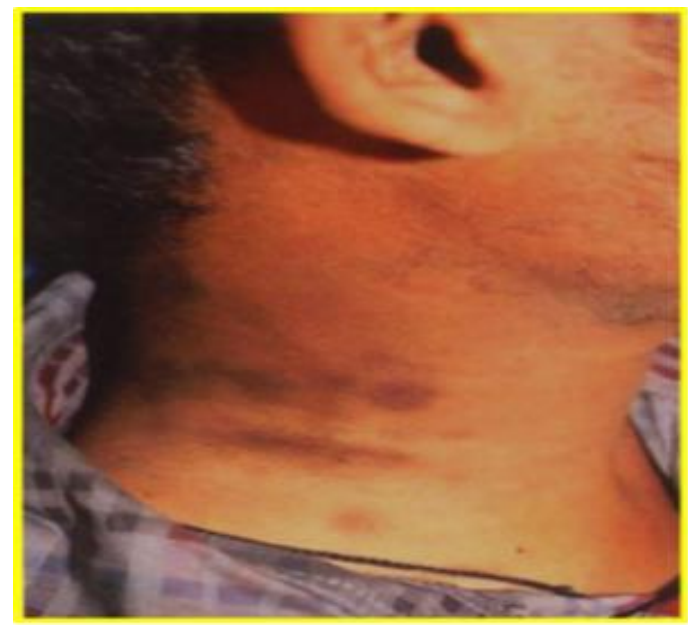

Fig-1: Pigmentation on neck.

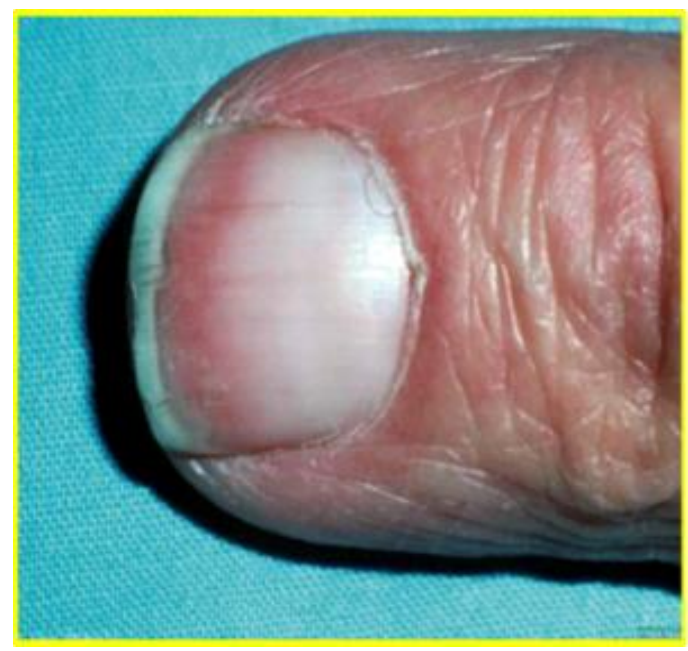

Fig-2: Nails presentation.

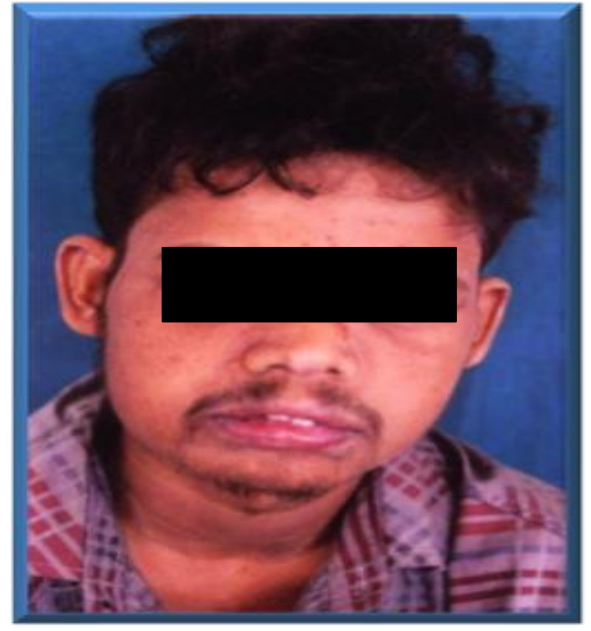

Fig-3: Extra-oral features.

On intraoral examination, there was generalised pallor of the oral mucosa. The upper labial mucosa revealed solitary, ill-defined, irregular in shape ulcer extending from the labial frenum till the posterior region. The floor was erythematous and was covered with pseudomembranous slough. On palpation it was tender (Fig.4). Similar ulcer seen on lower labial mucosa covering the entire labial mucosa (Fig.5). Right buccal mucosa revealed presence of diffuse area of encrustation extending from retro-commisural area till pterygomandibular region and from upper to lower buccal vestibule. It was covered with slough at some areas and hyperpigmentation was seen in the posterior region (Fig.6). Left buccal mucosa was greyish white in appearance (Fig.7). Palate appeared pale with similar ulceration with pseudomembranous slough seen at the posterior part of the hard palate and on the soft palate (Fig.8). Pallor noted on tongue dorsum. Ventero-lateral surfaces presented with diffuse areas of greyish white slough covering the erythematous ulcerations below it and surrounded by focal areas of diffuse blackish pigmentation (Fig.9). Along with this, his oral hygiene was also poor with generalized gingival inflammation and calculus deposits seen. Yellowish discoloration of teeth was noted in full complement of permanent dentition present.

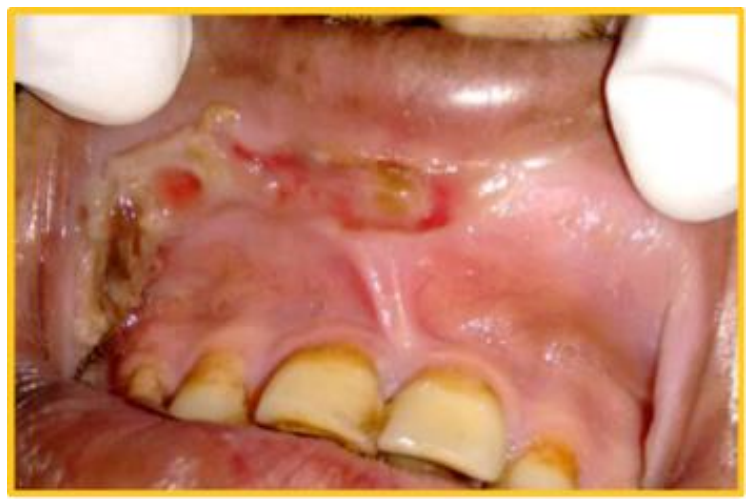

Fig-4: Ulceration on upper labial mucosa. 


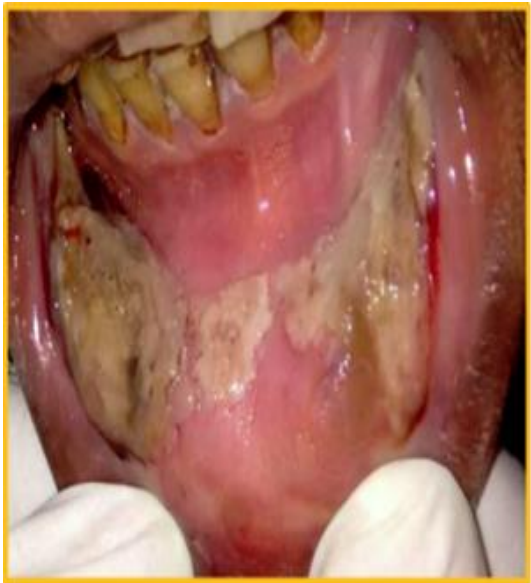

Fig-5: Ulceration on lower labial mucosa.

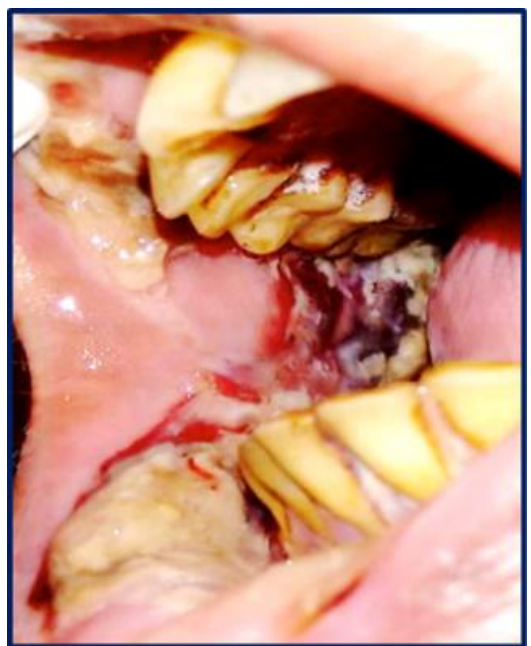

Fig-6: Ulceration on right buccal mucosa.

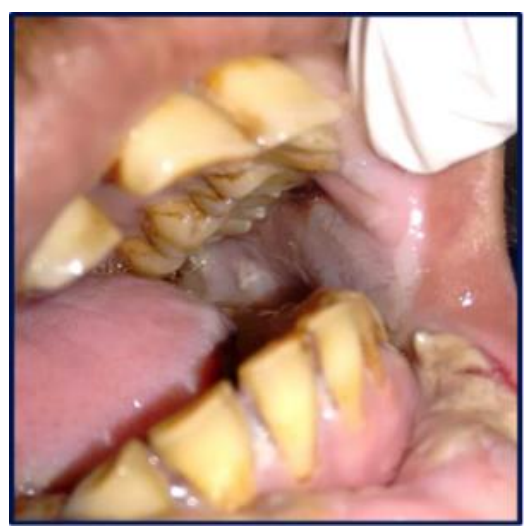

Fig-7: Left buccal mucosa.

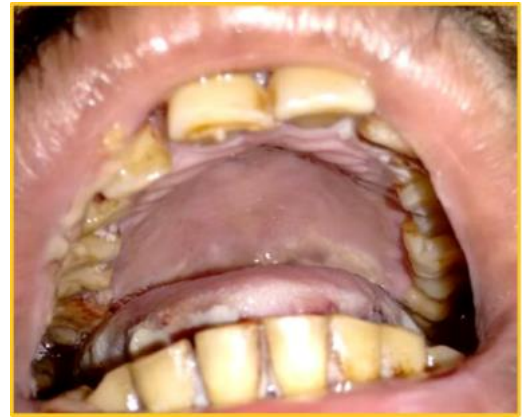

Fig-8: Palate.

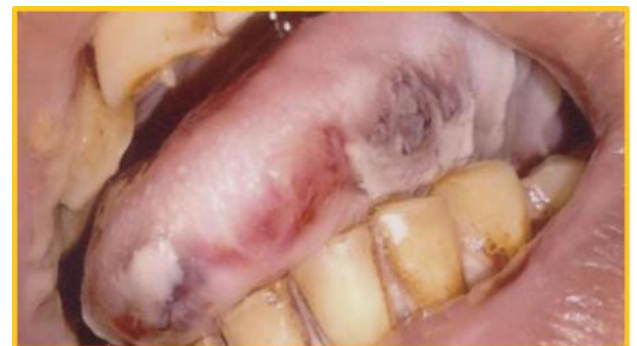

Fig- 9: Ulceration covered with pseudomembranous slough on tongue.

On the basis of chief complaint, history and clinical examination; a working diagnosis of acute multiple ulcers involving the buccal mucosae, tongue, labial mucosae and palate was given. The differentials were listed as Erythema Multiforme Major and Ulcerative Uremic Stomatitis. The hematology report revealed low heamoglobin. Peripheral smear reported Normocytic Hypochromic Anemia With Neutrophilic Leukocytosis And Thrombocytopenia.The serum chemistry report revealed increase in serum creatinine, blood urea nitrogen (BUN), serum potassium and reduced serum calcium and sodium.On OPG, full compliment of permanent dentition was present.Loss of lamina durawrt 36 and 37 and 47 was noted.Altered trabecular pattern was seen in the region of 36 and 37 (Fig.10).

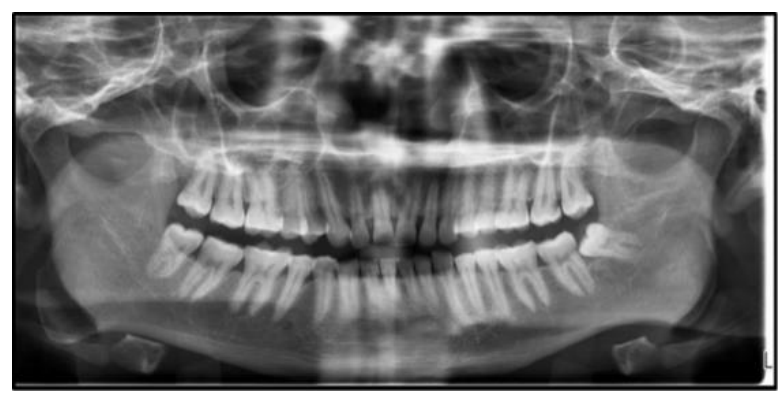

Fig-10: OPG.

Ultrasonography of abdomen revealed that both kidneys were shrunken in size with increased echostructure, no calculi/ mass, no hydronephrosis and corticomedullary differentiation not well made out; giving the impression of Grade III Nephropathy.Hence, final diagnosis was made as Ulcerative uremic stomatitis.Patient was prescribed $3 \%$ hydrogen peroxide $\left(\mathrm{H}_{2} \mathrm{O}_{2}\right)$ gargles $(1: 1$ in water) four times a day and Benzydamine hydrochloride oral rinse as rinse $15 \mathrm{~mL}$ in mouth (for 30 seconds, followed by expulsion from mouth) 3-4 times per day, as palliative care regime. Along with it, the patient was referred to the Nephrologist for management of underlying pathology.

\section{DISCUSSION \\ Uremic Stomatitis}

This condition is seen in patients with undiagnosed or untreated End Stage Renal Disease ${ }^{6}$. It was first mentioned by Lancereaux in 1887 and described by Barie in $1889^{7}$. 


\section{Epidemiology}

- Incidence of uremic stomatitis is very low.

- It occurs usually in advanced renal failure [5].

\section{Etiology}

It manifests due to raised levels of ammonia compound seen in CRF [8].

\section{Pathophysiology}

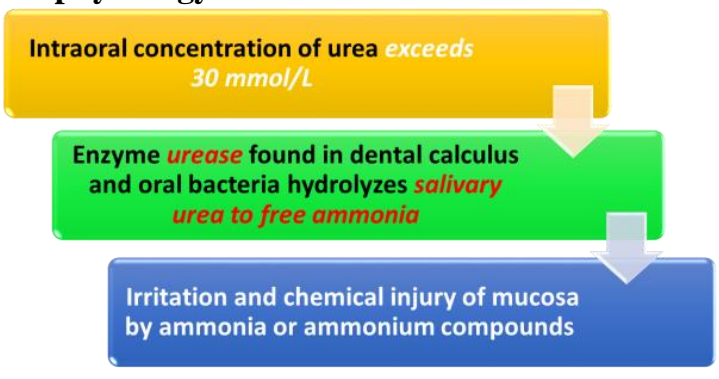

Hence, uremic stomatitis appears when blood urea levels $>30 \mathrm{mmol} / \mathrm{L}$.

Factors which aggravate this condition are:

- Poor oral hygiene

- Chronic gingivitis

- Periodontal disease

- Decayed teeth

- Xerostomia

- Smoking

Lesions develop in sites where mucosa comes in contact with teeth [9].

\section{Clinical Features}

It was first described as a red lesion on the oral mucosa covered with a pseudomembrane and as ulcerative forms. It mostly involves the dorsal, ventral, and lateral parts of the tongue, buccal and labial mucosa, or retro-molar areas[10].

\section{Forms : [11]}

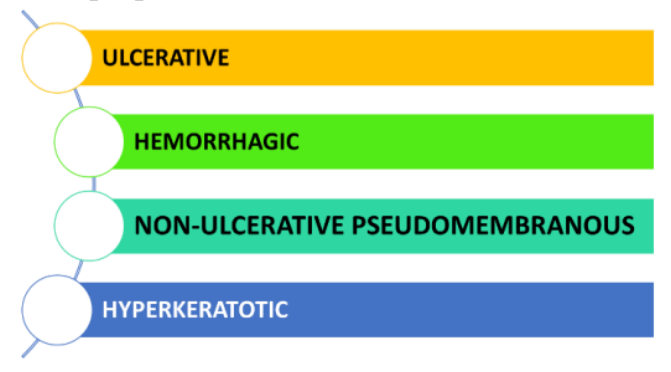

\section{Renal Osteodystrophy}

The changes manifesting in the bone are collectively termed as "renal osteodystrophy". The pathophysiology is as described in the diagram below (Fig. 11).[12]

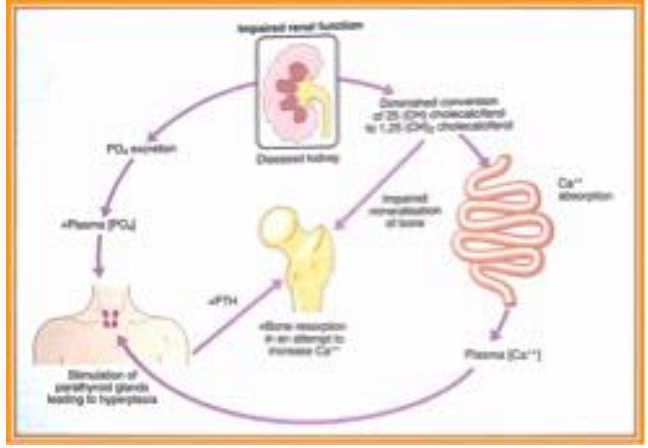

Fig-11: Pathophysiology of renal osteodystrophy.

It manifests as:

- Radiolucent giant cell lesions

- Maxillary brown tumors

- Enlargement of the skeletal base

- Metastatic soft-tissue calcification [13].

- Demineralization and Decreased trabeculation

- Loss of lamina dura

- Granular bone pattern

- 'Ground-glass'” appearance[14]

\section{Histopathology}

Histopathological signs of uremic stomatitis are not specific, the role of histopathology is to exclude other pathologic conditions [15].

\section{Management}

Intraoral lesions are resistant to local treatment as long as blood concentration of urea remains high. Hence the primary emphasis should beon the correction of the systemic pathology, supported by palliative oral care [16].

\section{Palliative Care}

- Scaling of the teeth to remove calculus deposits, which may contain urease.

- Hydrogen peroxide mouth rinses aid in elimination of anaerobic bacteria producing ammonia, to neutralize ammonia and the condition of acidosis.

- Oral manifestations persist usually for 2 to 3 weeks and may heal spontaneously with resolution of the underlying uremia and lowering of blood urea nitrogen (BUN) levels [17].

\section{CONCLUSION}

"The mouth is the mirror to the body", as rightly said by Sir William Osier. It is a well-known verity that many systemic diseases manifest in the oral cavity.Irrespective of the organ system involved, changes frequently occur in the oral cavity reflecting disease elsewhere in the body. So it is accurately said that oral cavity is the mirror of the general health and ideal management of such manifestations is by treating the underlying disease first, followed by providing local therapy. 


\section{REFERENCES}

1. Harrison, T. R., \& Fauci, A. S. Harrison's principles of internal medicine. 2008. Schistosomiasis and other trematode infection, 1266-1271.

2. Udayakumar, P., Balasubramanian, S., Ramalingam, K. S., Lakshmi, C., Srinivas, C. R., \& Mathew, A. C. (2006). Cutaneous manifestations in patients with chronic renal failure on hemodialysis. Indian Journal of Dermatology, Venereology, and Leprology, 72(2), 119.

3. Greenberg, M. S., Glick, M., \& Ship, J. A. (2008). Burket's oral medicine (Vol. 77). Hamilton: Bc Decker.

4. Kerr, A. R. (2001). Update on renal disease for the dental practitioner. Oral Surgery, Oral Medicine, Oral Pathology, Oral Radiology, and Endodontology, 92(1), 9-16.

5. Gupta, M., \& Gupta, M. (2015). Oral conditions in renal disorders and treatment considerations-A review for pediatric dentist. The Saudi dental journal, 27(3), 113-119.

6. Depner, T. A. (2001, July). Uremic toxicity: urea and beyond. In Seminars in dialysis (Vol. 14, No. 4, pp. 246-251). Boston, MA, USA: Blackwell Science Inc.

7. Mini, M. M., Prasad, T. S., \& Thomas, V. (2015). Uremic stomatitis: report of two cases. Oral Maxillofac. Pathol. J, 6(2), 636-8.

8. Cerveró, A. J., Bagán, J. V., Soriano, Y. J., \& Roda, R. P. (2008). Dental management in renal failure: patients on dialysis. Med Oral Patol Oral Cir Bucal, 13(7), E419-26.
9. de la Rosa García, E., Padilla, A. M., Romo, S. A., \& Ramírez, M. A. B. (2006). Oral mucosa symptoms, signs and lesions, in end stage renal disease and non-end stage renal disease diabetic patients. Medicina oral, patología oral y cirugía bucal. Ed. inglesa, 11(6), 3.

10. Carl W., Wood RH. (1976).The dental patient with chronic renal failure, Quintessence Int 7:9-15

11. Jaspers., Mark T. (1975).Unusual oral lesions in a uremic patient. Oral Surg Oral Med Oral Pathol; 39(6): 934-944.

12. Hruska, K. A., \& Teitelbaum, S. L. (1995). Renal osteodystrophy. New England Journal of Medicine, 333(3), 166-175.

13. Queiroz, S. M., Vasconcelos, R. G., Andrade, A. L. D. L. D., Amorim, A. G., Gordón-Nuñes, M. A., Freitas, R. D. A., \& Galvão, H. C. (2013). Maxillary brown tumor associated with chronic kidney failure: A case report. Jornal Brasileiro de Patologia e Medicina Laboratorial,49(6), 424428.

14. Antoniades, D. Z., Markopoulos, A. K., Balaskas, I., Patrikalou, E., \& Grekas, D. (2005). Ulcerative Uremic Stomatitis Associated with Untreated Chronic Renal Failure: Report of a Case. Oral Surgery, Oral Medicine, Oral Pathology, Oral Radiology and Endodontology, 2(100), 193.

15. Vanholder, R., Argiles, A., Baurmeister, U., Brunet, P., Clark, W., Cohen, G., ... \& Jörres, A. (2001). Uremic toxicity: present state of the art. The International journal of artificial organs, 24(10), 695-725.

16. Little. (2002). Dental management of medically compromised patients. $6^{\text {th }} \mathrm{Ed}$, 